University of New Hampshire

University of New Hampshire Scholars' Repository

Space Science Center

Institute for the Study of Earth, Oceans, and

Space (EOS)

$11-21-2000$

\title{
Analog processing of signals from a CZT strip detector with orthogonal coplanar anodes
}

\author{
K Larson \\ University of New Hampshire - Main Campus \\ V T. Jordanov \\ Digirad \\ Mark L. McConnell \\ University of New Hampshire - Main Campus, mark.mcconnell@unh.edu \\ John R. Macri \\ University of New Hampshire - Main Campus, John.Macri@unh.edu \\ James M. Ryan \\ University of New Hampshire, James.Ryan@unh.edu
}

See next page for additional authors

Follow this and additional works at: https://scholars.unh.edu/ssc

Part of the Astrophysics and Astronomy Commons

\section{Recommended Citation \\ Kipp Larson ; Valentin T. Jordanov ; Mark L. McConnell ; John R. Macri ; James M. Ryan ; Allen Drake ; Louis-Andre Hamel and Olivier Tousignant "Analog processing of signals from a CZT strip detector with orthogonal coplanar anodes", Proc. SPIE 4141, Hard X-Ray, Gamma-Ray, and Neutron Detector Physics II, 336 (November 21, 2000); doi:10.1117/12.407596; http://dx.doi.org/10.1117/12.407596}

This Conference Proceeding is brought to you for free and open access by the Institute for the Study of Earth, Oceans, and Space (EOS) at University of New Hampshire Scholars' Repository. It has been accepted for inclusion in Space Science Center by an authorized administrator of University of New Hampshire Scholars' Repository. For more information, please contact Scholarly.Communication@unh.edu. 


\section{Authors}

K Larson, V T. Jordanov, Mark L. McConnell, John R. Macri, James M. Ryan, A Drake, L A. Hamel, and O Tousignant 


\title{
Analog Processing of Signals from a CZT Strip Detector with Orthogonal Coplanar Anodes
}

\author{
K. Larson ${ }^{* a}$, V. Jordanov ${ }^{b}$, M. L. McConnell ${ }^{a}$, J.R. Macria, J.M. Ryan ${ }^{\mathrm{a}}$, A. Drake ${ }^{\mathrm{a}}$, \\ L.-A. Hamel ${ }^{\mathrm{c}}$, O. Tousignant ${ }^{\mathrm{c}}$

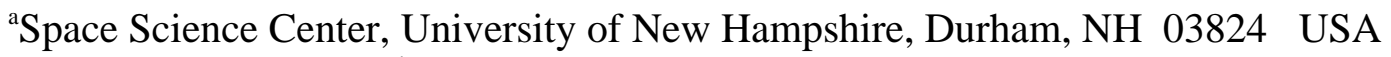 \\ ${ }^{b}$ Yantra, Durham, NH 03824 USA \\ 'University of Montreal
}

\begin{abstract}
We present the requirements, design, and performance of an analog circuit for processing the noncollecting anode strip signals from a cadmium zinc telluride (CZT) strip detector with orthogonal coplanar anodes. Detector signal simulations and measurements with a prototype are used to define the range of signal characteristics as a function of location of the gamma interaction in the detector. The signals from the non-collecting anode strip electrodes are used to define two of the three spatial coordinates including the depth of interaction, the $\mathrm{z}$ dimension. Analog signal processing options are discussed. A circuit to process the signals from the non-collecting anode strips and extract from them the depth of interaction is described. The circuit employs a time-over-threshold (TOT) measurement. The performance of the detector prototype with a preliminary version of this circuit is presented, and future development work is outlined.
\end{abstract}

Figure 3. CZT, imaging, gamma-ray, electronics

\section{INTRODUCTION}

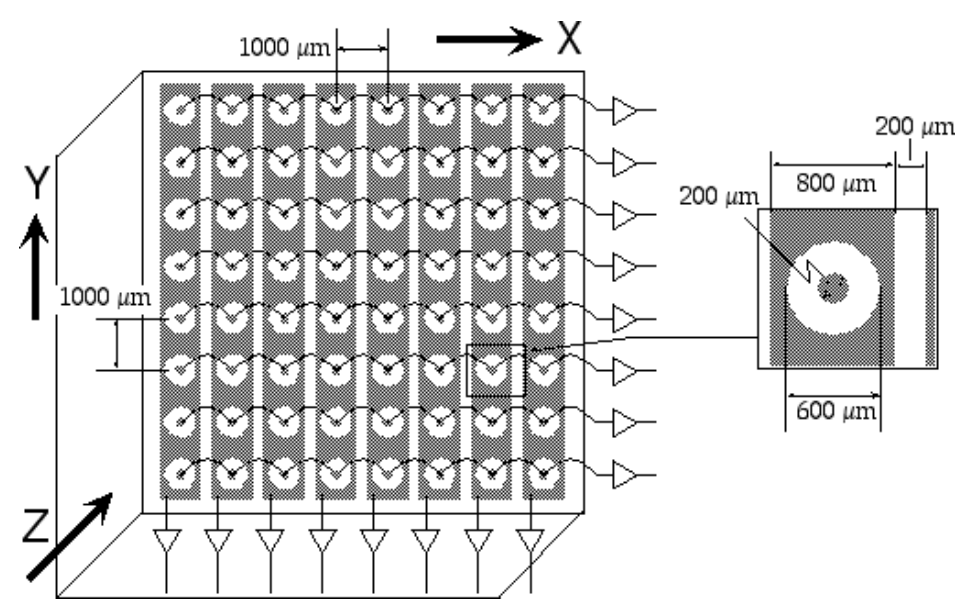

Figure 1. Schematic diagram showing the layout and read out of the orthogonal coplanar anode design. Strip columns (X) are read out on the bottom. Pixel rows (Y) are read out on the right. The gold contact pattern dimensions shown here in grey correspond to those of our prototype assemblies. Prototype CZT thickness is $5 \mathrm{~mm}$. A uniform cathode contact (not shown) is on the opposite surface $(\mathrm{Z}=0)$.
CZT strip detectors with coplanar anode geometries (Figure 1) have demonstrated both good energy and spatial resolution in two dimensions ${ }^{1}$. In imaging applications where photons are incident across a wide range of angles, knowledge of the third spatial dimension, z, the depth of interaction, is also desired. One such instrument application under development for astrophysical observations is TIGRE, the Tracking and Imaging Gamma Radiation Experiment ${ }^{2}$. The spatial resolution goal for these detectors in the TIGRE instrument is $1 \mathrm{~mm}$ in each of three dimensions. It has been shown $n^{1,3,4}$ that depth information may be obtained from measuring the relative pulse heights of the anode and cathode for an event. While this method provides sufficient $\mathrm{z}$ dimension resolution for TIGRE, it

\footnotetext{
*Correspondence: Email: klarson@ comptel.sr.unh.edu, John.Macri@unh.edu
} 
necessitates an electrical connection for signal processing to the cathode. This would interfere with fabrication of large closely packed arrays of imaging detector modules. For this reason, anode strip signal characteristics are being explored for a means of measuring the depth of interaction.

We are using a data acquisition system (DAQ) employing digital signal processing to measure and characterize these signals ${ }^{3}$. The digital DAQ provides a valuable tool for this study. While this digital approach yields good results in the laboratory, it is currently considered impractical for many-channel, lowpower space applications such as TIGRE. For this reason we are pursuing a low-power analog circuit solution.

Figure 1 illustrates the orthogonal coplanar anode contact geometry of the prototype detector. Signals from charge collecting anode pixels interconnected in rows are used to provide the energy measurements and the $\mathrm{Y}$ coordinate, and orthogonal non-collecting anode strips are used to provide the $\mathrm{X}$ coordinate. The circuit described here is designed to process the signals from the orthogonal strips and extract the $\mathrm{Z}$ coordinate of the gamma interaction.

\section{HISTORY OF DESIGN}

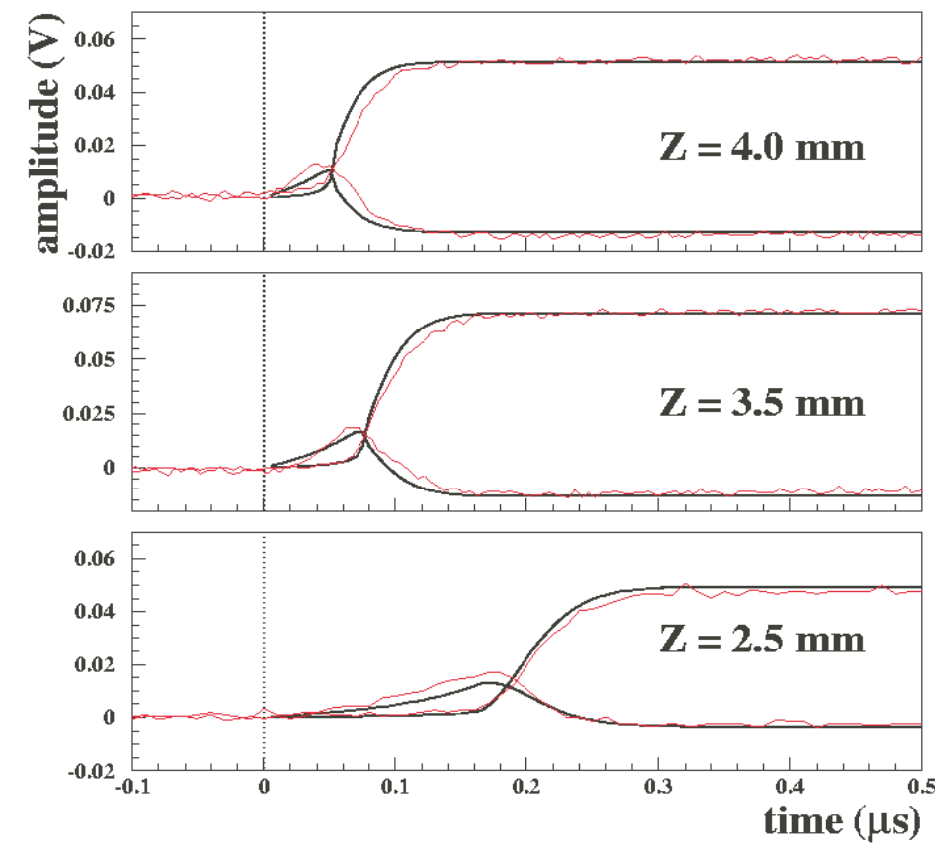

Figure 2. GEANT simulation of events at three different depths in $\mathrm{Z}$, with three measured events showing a strong match.
A signal simulation tool at the University of Montreal was used to examine strip and pixel signals specific to our detector. Figure 2 show simulated signals for three different depths of interaction, along with matching measurements taken from the detector. Note that while the pixel signals (rising only) remain essentially constant with depth, the strip signals (rising and falling) are seen to vary significantly.

A trade study was conducted which looked at three strip signal parameters which vary with depth: rise time, time-overthreshold (TOT), and residual. These signal parameters are illustrated in Figure 3. Using the simulation, these parameters were calculated at points on a $10 \times 10 \times 15$ grid evenly spaced

throughout the volume of a $1 \times 1 \times 5 \mathrm{~mm}$ unit cell (one "pixel"). A plot of these parameters versus depth for a point below the center of the pixel is given in Figure 4. As the figure shows, the residual is not linear across $\mathrm{Z}$ and changes very slowly across the first $3 \mathrm{~mm}$ of the detector. Both risetime and TOT are roughly linear in Z, but TOT has a greater slope. Assuming equal timing resolution, the TOT would therefore provide better depth resolution. Analog design concepts for both risetime and TOT were explored, and it was determined that the TOT would be the more simple implementation with fewer components. For these reasons our studies were focused on employing the TOT approach. 


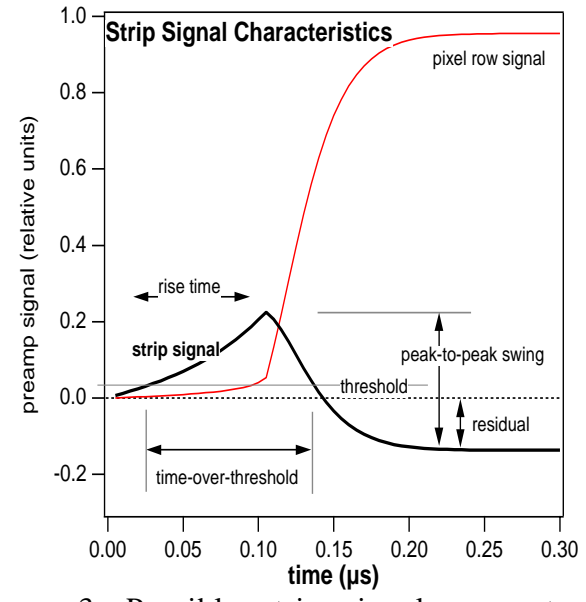

Figure 3. Possible strip signal parameters for extracting depth of interaction information.

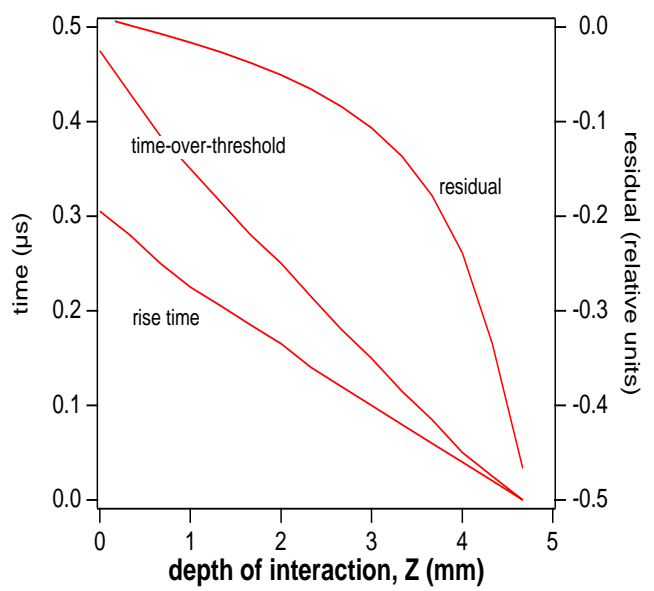

Figure 4. Parameters plotted vs depth for events in the center of the pixel.

\section{DESIGN SIMULATION}

Figure 5 shows a simplified schematic of the TOT to amplitude conversion circuit. The strip signal enters the voltage comparator, which puts out a logic zero as long as the input is above the threshold. The leading edge of this output triggers the one-shot, which puts out a logic zero for a specified amount of time, in this case 3usecs. This output keeps the MOSFET M1 switch closed for $3 \mu$ secs. At the same time, the JFET J1 is turned on and charges the $330 \mathrm{pF}$ capacitor for a time equal to the logic zero time of the comparator output. This time coresponds to the time-over-threshold. The capacitor holds the charge with the TOT information stored as a voltage until the MOSFET switch is opened by the return of the one-shot output to +5 Volts. This discharges the capacitor and allows the circuit output to return to zero, a self-resetting feature. A timing diagram of the comparator, one-shot, and TOT output can be seen in Figure 6.

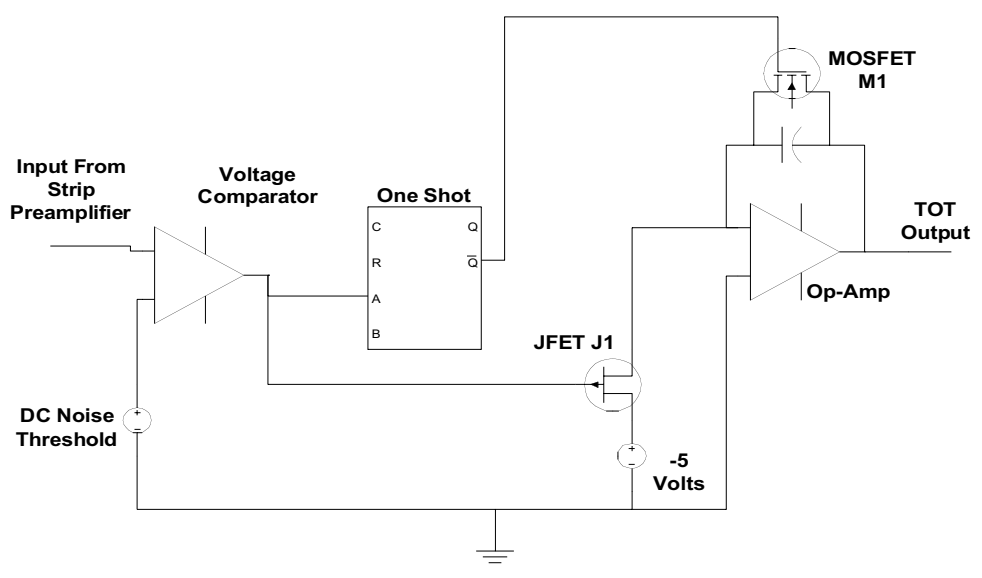

Figure 5. Simplified TOT circuit schematic. 


\section{Comparator Output}

One-Shot Output

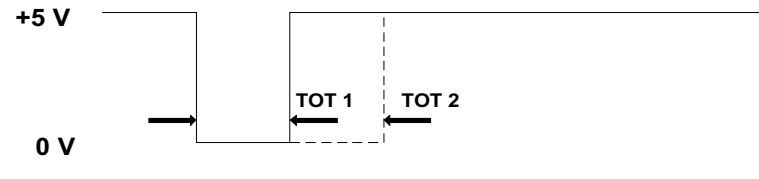

$0 \mathrm{~V}$

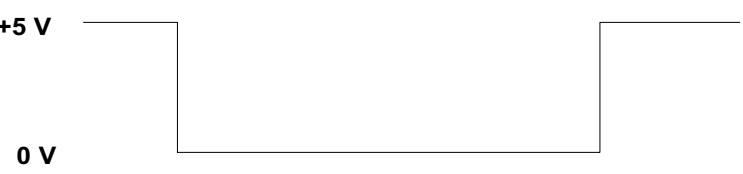

TOT Circuit Output

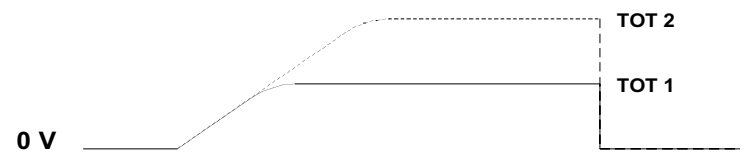

Figure 6. Relative timing of the comparator, one-shot, and TOT output. The length of the one-shot is preset, while the length of the comparator output varies with the strip signal time over threshold.

This circuit would be part of the front end electronics of a larger system which would simultaneously process both pixel and strip signals for all events. A possible configuration for such a system can be seen in Figure 7 with the TOT to amplitude circuit discussed here hilighted in darker grey. It would provide timeover-threshold information from the non-collecting anode strips in the form of a voltage amplitude to be read by an ADC.

A delay line or differential amplifier would be used to measure the peak to peak amplitude (see Figure 3). This would provide the trigger for external circuitry indicating that an event had occurred on that strip. Events with a small positive lobe may not trigger the TOT circuit, so this triggering method is necessary to allow triggering of events at any depth in the detector. Note that if the positive lobe of the strip signal does not trigger the TOT comparator, the TOT output will be zero, indicating that the gamma interaction occurred close to the anode.

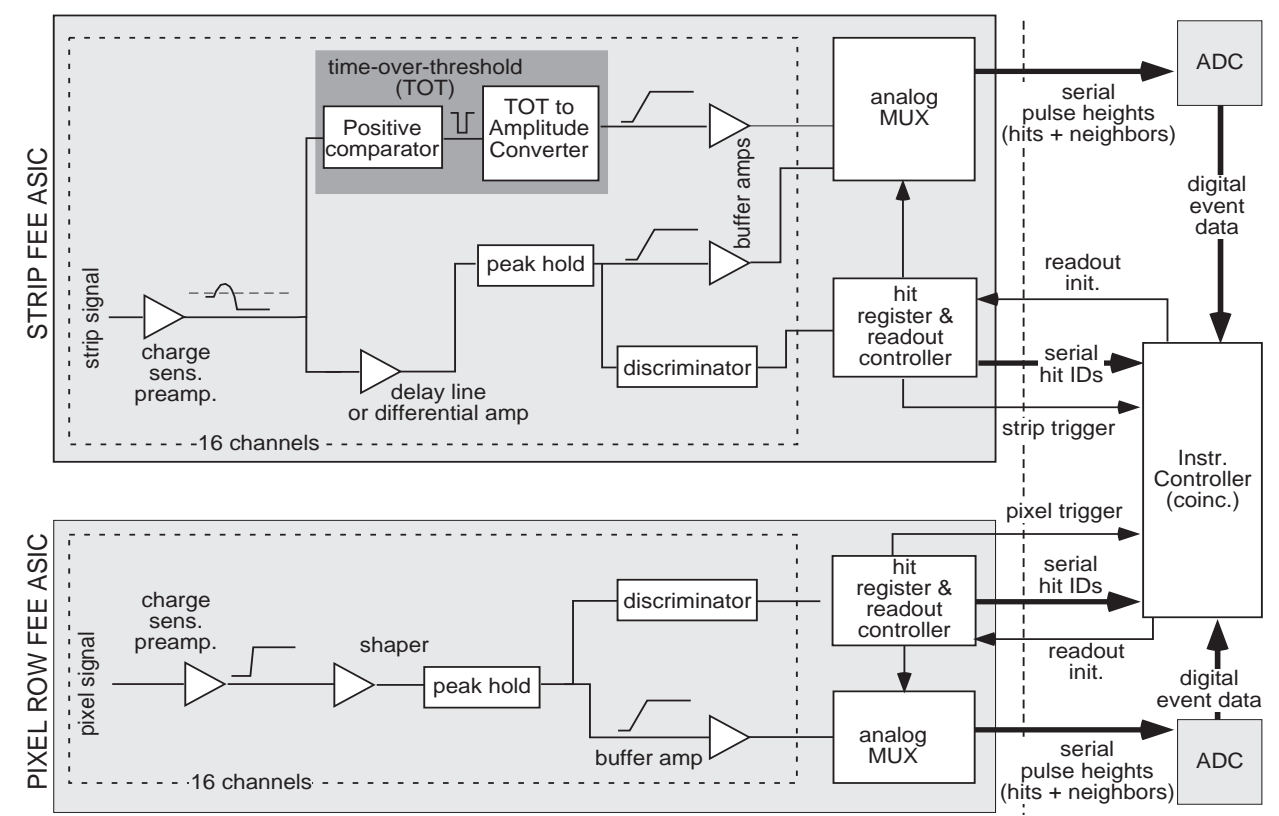

Figure 7. System design for front-end electronics for processing both pixel and strip signals. 


\section{PROTOTYPE TESTS}

A prototype of the circuit was laid out on vector board to facilitate design modifications. After setting the threshold to a level just above the preamplifier noise, measurements were made using $1.3 \mathrm{MeV}$ photons from a $\mathrm{Co}^{60}$ source. The detector was biased at -700 Volts, with the anode differential voltage set to 30 Volts. Events were measured on a digital storage oscilliscope, which was triggered from a pixel channel preamplifier output. Events were recorded which provided coincident pixel, cathode, strip, and TOT outputs. Figure 8 shows scope traces of two events, one (left) with a large anode to cathode ratio indicating an event nearer the anode, and the other (right) with a smaller anode to cathode ratio, indicating an event closer to the cathode. The increase in time over threshold for an event closer to the cathode is reflected in both the strip signal and TOT circuit output. The rounding of the leading edge of the TOT output is due to low pass filtering of the output to reduce noise.
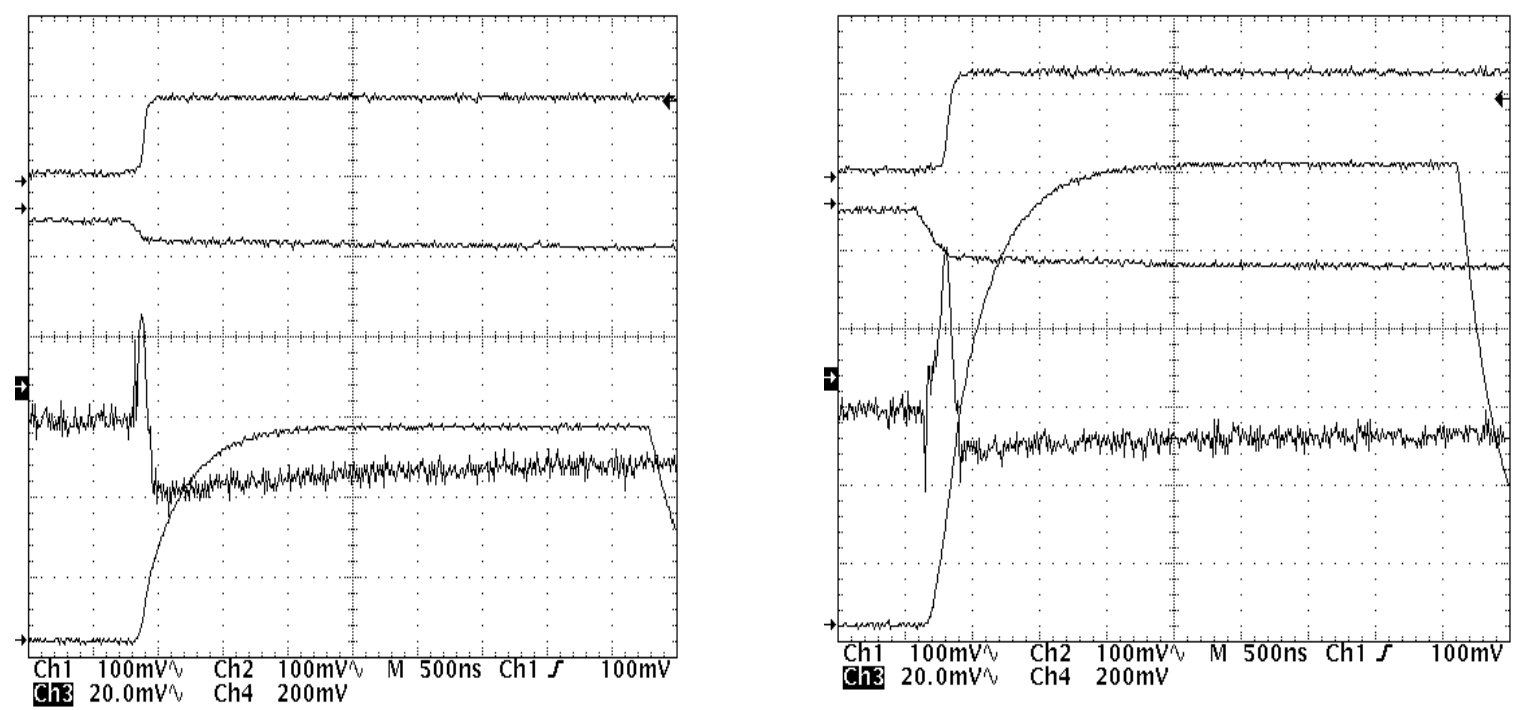

Figure 8. Digital storage oscilliscope trace of, from top, anode, cathode, strip preamplifier output, and TOT circuit output for $\mathrm{Co}^{60}$ events at two different depths.

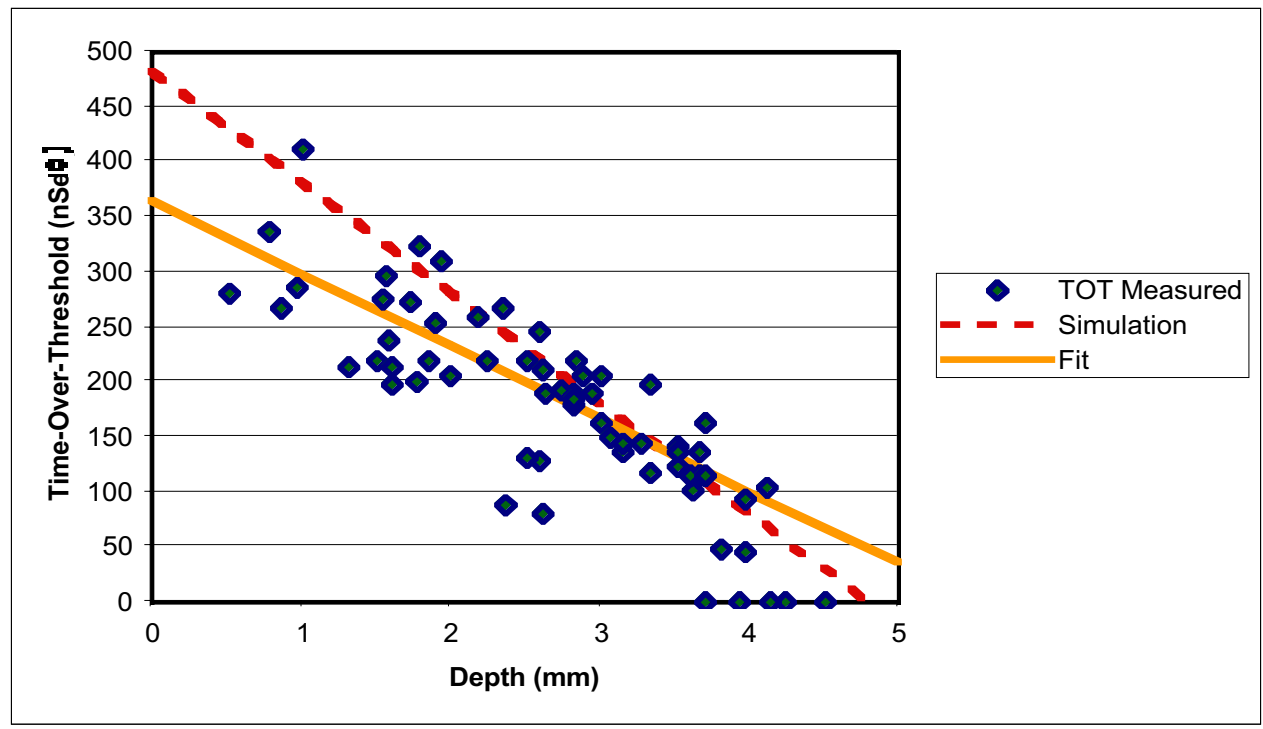

Figure 9. Plot of TOT measurements versus depth, with simulation results and least-squares fit. . 
A pulse generator was used to calibrate the TOT, and the formula depth $=(1$-cathode/anode $) * 5 \mathrm{~mm}$ was used to compute the depth, $\mathrm{z}$, from the anode and cathode pulse heights. The measurements on a sample of 60 events were then plotted in terms of TOT (ns) vs. depth $(\mathrm{mm})$, and compared with the simulation (Figure 9). The slope is not as steep as that of the simulation because the threshold of the circuit was slightly larger than that used in the simulation in order to minimize triggering on noise. The standard deviation is $.66 \mathrm{~mm}$, which translates to a spatial resolution of $1.55 \mathrm{~mm}$ FWHM. This is greater than the desired $1 \mathrm{~mm}$ resolution, but does demonstrate the usefulness of the technique. Several events were measured with a TOT output of zero. These are events which occurred close to the anode, but had a positive lobe too small to trigger the comparator. As mentioned above, such events could be recognized using a second comparator triggering on the negative lobe of the strip signal. It is expected that with a PCB layout the noise will be sufficiently low to get below $1 \mathrm{~mm}$ resolution

\section{CONCLUSIONS}

A circuit has been presented which determines the depth of interaction of gamma-ray events in a prototype CdZnTe coplanar-anode imaging detector by measuring the time-over-threshold of non-collecting anode strip signals. Motivations for using the TOT parameter to extract depth information were discussed, and circuit design considerations were examined. A spatial resolution of $1.55 \mathrm{~mm}$ was achieved using a prototype of the TOT circuit. Although limited by the signal to noise performance of the first vector board prototype circuit, the results match closely with simulation data, and demonstrate the usefulness of this technique for future $\mathrm{CdZnTe}$ instruments which require three dimensional spatial information.

\section{FUTURE WORK}

Additional noise reduction with the vector board circuit is planned, followed by a PCB implementation of the circuit for all detector channels. A finely collimated gamma beam will be used to facilitate the study of circuit performance for interactions at any location in the detector. An automated system will be used in the laboratory to acquire event data providing a better tool to evaluate position resolution and detector efficiency performance. A circuit will be developed which will allow the relative pulse heights of a strip and its neighbors to be compared for the purpose of interpolating the $\mathrm{X}$ dimension position of an event to better than the pitch of the strips. One possible method to accomplish this would be through the measurement of the relative peak-to-peak strip signal values (see Figure 3).

\section{ACKNOWLEDGEMENTS}

The authors wish to thank Todd Felege and Mickel McLish for their help in obtaining these measurements. This work is supported by NASA's High Energy Astrophysics Gamma Ray Astronomy and Analysis program and by the Natural Sciences and Engineering Research Council (NSERC) or Canada.

\section{REFERENCES}

1. O. Tousignant, L. A. Hamel, P. Dufor, F. Joly, J. R. Macri, M. L. McConnell, J. M. Ryan, and V. T. Jordanov, "Energy and position resolution of a CdZnTe gamma-ray detector with orthogonal coplanar anodes." Proc. SPIE, 3768, pp. 38-48, 1999.

2. D. Bhattacharya, D.D. Dixon, V. Kong, T.J. O'Neill, R.S. White, A.D. Zych, J.Ryan, M. McConnell, J. Macri, J. Samimi, A. Akyuz, W.A. Mahoney, L.S. Varnell, "A Tracking and Imaging Gamma-Ray Telescope (TIGRE) for Energies of 0.3 to $100 \mathrm{MeV}$,” Proc. 26th Internat. Cosmic Ray Conf., Salt Lake City, 5, pp. 72-75, 1999.

3. M.L McConnell, J.R. Macri, J.M.Ryan, K. Larson, L.-A. Hamel, G. Bernard, C. Polerleau, O. Tousignant, J.-C. Leroux, and V. Jordanov, "Three-dimensional imaging and detection efficiency performance of orthogonal coplanar CZT strip detectors," to be published in Proc. SPIE 4121, 2000.

4. Z. He, W. Li, G. F. Knoll, D. K. Wehe, J. Berry, and C. M. Stahle, "3-D position sensitive CdZnTe gamma-ray spectrometers," NIM, A422, pp. 173-178, 1999. 